Document downloaded from:

http://hdl.handle.net/10251/61026

This paper must be cited as:

Quesada, R.; Burdío, F.; Iglesias, M.; Dorcaratto, D.; Cáceres, M.; Andaluz, A.; Poves, I.... (2014). Radiofrequency pancreatic ablation and section of the main pancreatic duct does not lead to necrotizing pancreatitis. Pancreas. 43(6):931-937. doi:10.1097/MPA.0000000000000156.

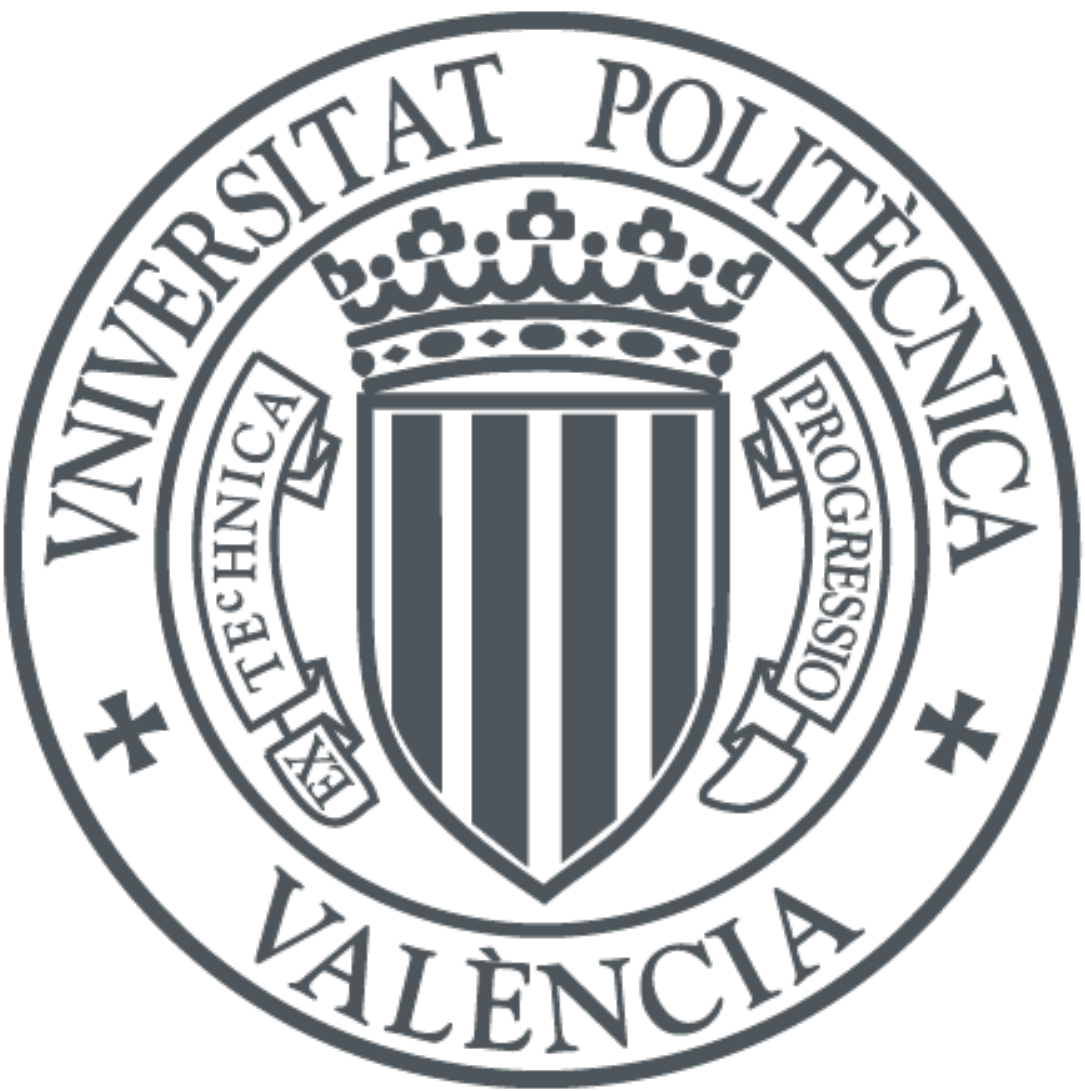

The final publication is available at

http://dx.doi.org/10.1097/MPA.0000000000000156

Copyright Lippincott, Williams \&amp;amp; Wilkins

Additional Information 


\section{Radiofrequency pancreatic ablation and section of the main pancreatic duct does not lead to necrotizing pancreatitis}

Rita Quesada, MS, ${ }^{1}$ Fernando Burdío, PhD, ${ }^{2}$ Mar Iglesias, PhD, ${ }^{3}$ Dimitri Dorcaratto, $\mathrm{PhD},{ }^{1}$ Marta Cáceres, MD, ${ }^{2}$ Anna Andaluz, PhD,${ }^{4}$ Ignasi Poves, PhD, ${ }^{2}$ Tomás Castiella, $\mathrm{PhD},{ }^{5}$ Patricia Sánchez, MD, ${ }^{2}$ Enrique Berjano, PhD, ${ }^{6}$ Luis Grande, PhD. ${ }^{2}$

From the "Instituto Municipal de Investigación Médica", 'Barcelona, Spain; Department of Surgery, ${ }^{2}$ Hospital del Mar, Barcelona, Spain; Department of Pathology, ${ }^{3}$ Hospital del Mar, Barcelona, Spain; Department of Animal Surgery, ${ }^{4}$ Veterinary Faculty, University of Barcelona, Spain; Department of Pathology, ${ }^{5}$ Hospital Clínico Universitario « Lozano Blesa », Zaragoza; Department of Electronic Engineering, ${ }^{6}$ Polytechnic University of Valencia, Spain.

Address reprint requests and correspondence to:

Fernando Burdío, MD.

Servicio de Cirugía. Hospital del Mar. Passeig Maritim 25. 08003 Barcelona, Spain

Tf: +349324833490

FAX: +34932483433

E-mail: fburdio@hotmail.com

RF ablation and section of the pancreatic duct.

Disclosures: F. Burdío, E. Berjano and R. Quesada declare stock ownership in Apeiron Medical S.L., a company that has a license for the patent US 8.303.584.B2, on which the device have been employed. The rest of the authors have no conflict of interests or financial ties to disclose. 
Objective: Determine whether Radiofrequency ablation (RFA) of the pancreas and subsequent transection of the main pancreatic duct may avoid the risk of both necrotizing pancreatitis and postoperative pancreatic fistula (POPF) formation.

Methods: Thirty-two rats were subjected to RFA and section of the pancreas over their portal vein. Animals were killed at 3, 7, 15 and 21 days (Groups 0-3, respectively). Two additional control groups (sham operation and user manipulation only, respectively) of 15 days of postoperative period were considered. Postoperative complications, histological changes (including morphometric and immunohistochemical analysis) and incidence of POPF were evaluated.

Results: A significant increase in serum amylase levels $(p<0.05)$ on the $3^{\text {rd }}$ postoperative day which return to baseline levels in the following weeks was noted in groups 0-3. Those groups showed a rapid atrophy of the distal pancreas by apoptosis with no signs of necrotizing pancreatitis or POPF. The distal pancreas in groups 1-3 compared to group 0 and control groups showed a significant increase of small islets $\left(<1000 \mu \mathrm{m}^{2}\right)$.

Conclusions: The rapid acinar atrophy of the distal pancreas after RFA and section of the pancreatic ducts in this model does not lead to necrotizing pancreatitis.

Key words: radiofrequency ablation, necrotizing pancreatitis, apoptosis, atrophy, main pancreatic duct, duct ligation.

Original article

\section{Introduction}

It is well known that after ligation of the rat pancreas, acinar cells disappear from the distal gland in 5 days with no signs of necrotizing pancreatitis ${ }^{1,2}$ probably because apoptosis of the acinar cells is the leading phenomenon after a completely obstructed main pancreatic duct ${ }^{1}$. However, even though the absence of an anastomosis of the pancreatic remnant may prevent many postoperative complications, the occlusion of the pancreatic stump with synthetic/biological substances or sutures has not been widely accepted due to a high percentage of pancreatic fistula (sometimes more than 
$50 \%)$ and postoperative collections ${ }^{3}$. Additionally, an increase risk of exocrine ${ }^{4}$ and endocrine pancreatic insufficiency cannot be ruled out ${ }^{5}$. In any case, the risk of a longlasting fistula formation is the more cumbersome complication after ligation of the main pancreatic duct after pancreaticoduodenectomy (PD) ${ }^{6}$.

Radiofrequency (RF) ablation is a local ablative method that can destroy tumors by thermal denaturation of proteins and has been used successfully in the treatment of other unresectable tumors in the liver, lung, kidney and bones ${ }^{7}$. Concerning the pancreas, given that most patients with pancreatic cancer have locally unresectable disease at the time of diagnosis with limited treatment options, there is a growing interest in this relatively new technique ${ }^{8}$. Additionally, RF energy has also been used both experimentally and clinically to manage the pancreatic remnant after distal pancreatectomies because of its ability to seal the main and secondary pancreatic ducts $^{9,10}$, but to our knowledge, it has not been used in other pancreatic interventions. In recent years, our group has tested the ability of an internally cooled RF electrode to seal vessels in the liver ${ }^{11,12}$ and in the kidney ${ }^{13}$. Preliminary studies using a porcine pancreatic model ${ }^{14}$ suggested that distal pancreatic transection by such systems can adequately seal the main pancreatic duct easily and safely. However, little is known about the use of RF ablation in the pancreas, although some studies suggest that its standard use is not recommended due to severe complications such as necrotizing pancreatitis $^{15}$.

With the above considerations in mind, our hypothesis is that, due to its ability to adequately seal the main pancreatic duct in the remnant pancreas, RF ablation of the pancreas and subsequent transection of the main pancreatic duct may avoid the risk of both necrotizing pancreatitis and the formation of pancreatic fistula.

\section{Materials and methods}

Animals 
A total of 39 female 6-week old Sprague-Dawley rats (Charles River Laboratories, Inc., Wilmington, MA, USA) were used for the study. Three rats were previously studied to set up the surgical approximation and were not considered in our analysis. The animal research protocol was approved by the Ethics Committee for Animal Experimentation of the PRBB (Barcelona Biomedical Research Park) and by the Government of Catalonia's Animal Care Committee (FBP-10-1280 y DMAH 5452).

\section{Experimental groups}

Six groups were considered: in groups 0 to $3(n=8$, each) RF pancreatic ablation and subsequent section of the whole pancreas was performed at the junction of the duodenal and gastrosplenic portions of the pancreas (over the portal vein) according to the anatomic rat nomenclature described by Richard et al. ${ }^{16}$.

The animals were killed at 3, 7, 15 and 21 days after the procedure, considered as Group 0 to 3, respectively. Additionally, two controls group of 15 days of postoperative period (PO) were considered: 1 ) Group $4(n=3)$ where animals were subjected to sham operation which consists on performing the surgical manipulation without ablation and section of the pancreas and 2) Group $5(n=4)$ where animals were subjected to user manipulation only without any surgical procedure. This group was considered to evaluate possible histological changes in the proximal and distal pancreas because of the pancreatic dissection and manipulation.

\section{Preoperative care}

All the animals were maintained on a commercial pellet food and water ad libitum under controlled environmental conditions for a week to avoid the influence of stress. The animals were weighed and initially sedated subcutaneously with buprenorphine $(0.001 \mathrm{mg} / \mathrm{kg}) 30 \mathrm{~min}$ before the procedure. Anaesthetic induction was performed with isoflurane at $4 \%$ and maintained at $2.5 \%$ in $100 \%$ oxygen. The plantar 
and palpebral reflexes were checked previous to the surgical procedure, and breathing was monitored during the procedure to increase the anaesthetic dose as required.

\section{Surgical technique}

All the surgical procedures were performed by the same surgical team (R.Q and F.B.). Thirty-two animals (groups $0-3$ ) underwent a pancreatic transection assisted by $\mathrm{RF}$ in the following manner. The peritoneum was entered through a midline laparotomy and the stomach, pancreas and spleen were mobilized. The spleen was retracted laterally, and the splenic vessels were dissected from the pancreatic tissue to prevent devascularisation of the pancreatic parenchyma. The pancreas of the experimental groups 0 to 3 was ablated and subsequently transected using the 3-mm version of the Coolinside ${ }^{\circledR}$ device (Apeiron Medical, Valencia, Spain). This device consists of a handheld instrument that performs a RF ablation with a cool-tip electrode of $20 \mathrm{~mm}$ length and permits section of the tissue with the built-in blade ${ }^{11-14}$. The pancreatic duct was not identified during the transection. The proximal and distal pancreas and the other viscera were replaced in anatomic position, and the incision was closed without abdominal drain in two layers using braided polyglycolic acid suture (4/0). The animals were allowed to recover from anaesthesia on an electric blanket and returned to their respective cages after recovering their reflexes. For control, 3 animals were subjected to sham operations (Group 4) in which the abdomen was opened, the internal organs moved, but the pancreas was not transected. Group 5 was not subjected to any surgical technique.

The ablation was standardized has follows: the prove was directly placed at the junction of the duodenal and gastrosplenic portions of the pancreas which is placed over the portal vein (according to the nomenclature of rat pancreas described by Richard, et al. $\left.{ }^{16}\right)$. The pancreas was mobilized and slightly tractioned to ensure a 5-mm minimum safe distance between RFA site and major peripancreatic vessels and surrounding viscera to avoid unexpected injuries. Radiofrequency energy was applied 
by the tip of the device until a full-thickness coagulation of the pancreas at that junction was achieved. This coagulated tissue was subsequently sectioned in the middle of the RFA. In all the procedures, ablation time, tissue impedance, current and power was recorded.

\section{Postoperative care and necropsy}

During PO, the animals had free access to food and water containing antibiotics for the firsts three days (Enrofloxacin, $500 \mathrm{mg} / \mathrm{kg}$ ). Buprenorphine $(0.01 \mathrm{mg} / \mathrm{kg}$ ) was administered subcutaneously as an analgesic therapy, every 12 hours for 3 days. Animals were monitored for physical activity, grooming routines, level of alertness, response to stimulation and other signs of stress with a condition score system previously approved by the Welfare committee. Serum amylase was obtained from the saphenous vein following the previous narcosis protocol described in all the animals before the initial incision and on postoperative days 3, 7, 15 and 21 as appropriate, with the aim of evaluating any possible sign of pancreatitis.

At the necropsy, the animals were euthanatized by $\mathrm{CO}_{2}$ inhalation at $100>x>70 \%$ concentration and a lateral laparotomy was carried out to explore the abdominal cavity. The presence of pancreatic fistula, abscess or abdominal fluid was recorded. Finally, the pancreas was extracted for histopathologic study.

\section{Reagents}

Polyclonal antibodies against the cleaved-caspase 3 (Asp175) and insulin (C27C9) were from Cell Signalling Technology (Danvers, STATE, USA). Anticytokeratin 7 antibody (RCK105) was from Santa Cruz Biotechnology (City CA, USA) and the secondary antibody was a avidin-biotin complex-conjugated solution of Real DAKO (EnVision, Copenhage, Denmark). 
The distal and the proximal pancreas were fixed in $10 \%$ paraformaldehyde immediately after its extraction. Fixed samples were embedded in paraffin, and sections of $3 \mu \mathrm{m}$ were stained with hematoxylin and eosin. Microscopic findings of coagulative necrosis were recorded, such as cytoplasmic shrinkage, densification and variable degree of chromatin condensation with focal formation of apoptotic-like bodies, along with the presence of abscesses to evaluate the histological changes of the pancreas after the surgical procedure. The proximal pancreas was used as a control of normal pancreatic tissue within subjects. Group 4 (sham) and 5 (negative control) were used to evaluate any histological differences due to the surgical technique and differences between subjects, respectively.

According to previous studies ${ }^{17-19}$ to determine the apoptotic response and the ductal proliferation of the pancreas, cleaved-caspase 3 (as an apoptosis marker) and cytokeratin 7 (as a duct cell marker) were analyzed in the most representative samples. Furthermore, immunohistochemical analysis of insulin was carried out in the same samples to evaluate the endocrine function of the distal and proximal pancreas. Sections $(3 \mu \mathrm{m})$ were fixed to APS-coated glass sides, dewaxed and rehydrated. After antigen retrieving with sodium citrate $0.01 \mathrm{M}$ at, $\mathrm{pH} 6$ and washes with TBS $\mathrm{x} 1$, the quenching of endogenous peroxidase activity was performed using $10 \% \mathrm{H}_{2} \mathrm{O}_{2}$ for 10 min. The slides were then blocked in $1 \%$ BSA, 1\% FBS in TBS $x 1$ for 10 min and treated with primary antibody overnight at $4^{\circ} \mathrm{C}$. Primary antibodies and dilutions were as follows: rabbit anti-cleaved caspase 3, 1:1600; rabbit anti-insulin, 1:400 and mouse anti-cytokeratin 7, 1:150. After washing in TBS $1 \mathrm{x}$ and TBS-Tween 1x, rabbit/mouse secondary antibody was applied for $45 \mathrm{~min}$ at room temperature. 3,3'Diaminobenzidine (DAB) chromogen was then applied, and the slides were counterstained with hematoxylin.

\section{Analyzed variables}


The primary outcomes of the study were (1) postoperative complications, such as necrotizing pancreatitis and other perioperative parameters and (2) the histological changes of the pancreatic tissue after RF-assisted transection including the histological preservation of the endocrine distal pancreas.

The secondary outcome of the present study was to analyze the occurrence of postoperative pancreatic fistulas (POPF), defined as any measurable volume of fluid on or after postoperative day 3 with an amylase content greater than 3 times the serum amylase activity (usually any undrained amylase-rich fluid collections/abscess).

\section{Morphometric analysis}

Computer-assisted measurements (Cell^ $\mathrm{B}$ image acquisition, Olympus, Tokyo, Japan) of insulin stained islets in sections from duodenal pancreas (proximal) and splenic pancreas (distal) were carried out at different time points according to previously described methods ${ }^{20,21}$. Four to eight immunohistochemically stained insulin slices per specimen were used for the morphometric analysis, 5 sections were chosen at random from each region (proximal and distal). A total of 501 islets (with a range of 20-83 islets from each specimen) were examined at x 100 magnification to estimate:

(1) Profile diameter of the islet "D" calculated from $\sqrt{a b}$ which corresponds to the major "a" and minor "b" diameter, respectively, ${ }^{20}$ (2) Mean hormonoreactive area "A" measured in $\mu \mathrm{m}^{2},{ }^{21}$ (3) Cell count of small islets and larger islets in proximal and distal rat pancreas at different time points, considering an empirical distinction between small islets (individual beta cells and islet-clusters) with a profile area of less than 1000 $\left.\mu m^{2}\right)^{21}$.

Consecutive immunohistochemically stained cleaved-caspase 3 slices were used for the quantitative analysis of positive cells using ImageJ v1.43m software (http://rsb.info.nih.gov/ij). The analysis was performed in 5 sections choose at random from each region (proximal and distal). Positive cleaved-caspase 3 cells were detected 
by applying a threshold to the image, separating ground signal from background signal. Circular objects were defined as contiguous set of pixels applying morphological operations to improve the resulting object quality ${ }^{22,23}$. Quantitative data were exported to statistical software for further analysis.

\section{Statistical analysis}

Data are presented as median and standard deviation. Main outcomes were compared and correlated between groups. The Kolmogorov-Smirnov test was used to determine whether values followed a normal distribution and the Levene to evaluate equality. Mean values of numerical data were compared through the Friedman test, the Mann-Whitney U test, the Kruskal Wallis test or ANOVA when appropriate. Additionally, non-linear fits (i.e. higher order regression models) and linear regression models were performed in order to determine the best fit of the apoptotic response. The goodness of fit of a model was assessed by Pearson correlation coefficient ( $r$ ), which was provided when any significance was detected. Differences in variables were considered to be significant at a threshold of $p<0.05$. Statistical analyses were performed with statistical software (SPSS version 19.0, SPSS, Inc., Chicago, IL, USA).

\section{Results}

\section{Survival studies and technical details}

There were no deviations from the protocol described in the methodology. Radiofrequency energy was applied during a mean ablation time of $89 \pm 50 \mathrm{~s}$ at a mean delivered power of $20 \pm 0.6 \mathrm{~W}$ (mean total delivered energy per ablation of $320 \pm$ $80 \mathrm{~J})$.

There were no major intraoperative complications or deaths. All the animals tolerated the procedure well and quickly recovered and ambulated. There were no signs of relevant disease in the postoperative follow-up, although in the immediate postoperative period the animals showed signs of stress and discomfort such as 
bristling hair or lack of grooming, as expected in a normal postoperative context. During the postoperative period the animals were active and showed no clinical symptoms of pancreatic disease, tolerating intake and had a growth curve with no significant differences other than the expected (preoperative weight $197.9 \pm 19.0 \mathrm{~g}$, mean prenecropsy weight at 3 weeks $251.6 \pm 17.1 \mathrm{~g})$.

Neither undrained amylase-rich fluid collections nor abscesses were observed in gross examination during necropsy and no clinical anatomic evidence of pancreatic fistula was observed in any case.

\section{Biochemical determinations}

Figure 1 shows serum amylase before surgery and on postoperative days 3, 7, 15 and 21. A significant increase in serum amylase levels $(p<0.05)$ on the 3rd day PO over their preoperative value with no clinical repercussions was noted in groups $0-3$, which returned to levels similar to baseline in the following weeks. Likewise, similar significant differences were found in serum amylase levels on the $3^{\text {rd }}$ day PO between groups 0-3 and groups 4 and 5 (Figure 1). When plotted the serum amylase levels against their preoperative serum amylase values, 7 animals (21\%) had levels above three times this level, but none of them presented clinical complications during the postoperative follow up and no intra-abdominal collections, signs of peritonitis or other complications were found during necropsy.

\section{Histopathological Findings}

The histopathological study distinguished between the three different areas in the animals subjected to radiofrequency ablation and pancreas transection: (1) the transection margin, (2) the proximal pancreas and (3) the distal pancreas

1) The transection margin of the pancreatic stump was macroscopically characterized by a yellow dehydrated area (Figure 2) and common histological pattern with a central area of coagulative necrosis $(\mathrm{Cn})$ surrounded by 
inflammatory infiltrate which varies in accordance with the day of necropsy. The ablative necrosis area at the transection margin was characterized by a mean maximum diameter of $5091 \pm 1654 \mu \mathrm{m}$ and a mean minimum diameter of 2434 $\pm 708 \mu \mathrm{m}$ for groups $0-3$. On the 3rd day, the inflammatory component was based on polymorphonuclear leucocytes (acute inflammation) and in some sections vascular congestion and slight oedema was also seen. The acute component decreases when the necropsy was carried out on days 15 and 21 after transection (Groups 2 and 3 ) changing to a granulomatous inflammatory infiltrate, with macrophages and multinucleated giant cells. Intense fibrosis was also present delimiting the necrotic material (see Figure 2 b).

2) The lobular architecture of the proximal pancreas appeared unchanged in all the animals, with total preservation of the endocrine and exocrine tissue (see Figure 3 a-d).

3) The distal pancreas showed progressive acinar cell disappearance with different stages over the postoperative period. By the end of the $3^{\text {rd }}$ day the total volume of acini appeared to be reduced, with loss of nuclear polarity and decreased zimogen in the cytoplasm. Apoptotic bodies were visible in most acinar units (see Figure $3 \mathrm{e}$ ). A week after the transection, there was a complete replacement of exocrine acini by pseudo-ductal complexes, based on apparently novel duct-like structures composed of low-cuboidal cells. The interlobular connective tissue contracted and adjacent lobules tended to become fused together (see Figure 3 i). On days 15 and 21 there were also scattered infiltrates of lymphocytes and plasma cells in the fibrous boundary. There also seemed to be an extension of adipose tissue surrounding the pancreatic tissue from the end of the $2^{\text {nd }}$ week (Figures $3 \mathrm{~m}, 3 \mathrm{q}$ ).

No histological changes were observed in endocrine or exocrine tissue of the control groups. During the histological study, no purulent inflammatory infiltrate was observed, but there were 3 micro-abscesses (less than $1000 \mu \mathrm{m}^{2}$ ) in three animals; two 
cases in Group 2 and one in Group 3. No other inflammatory components were found in any other animals. All the animals experienced an uneventful postoperative periods.

\section{Immunohistochemical analysis}

Immunohistochemical analysis of cleaved-caspase 3 showed isolated positive stain in the proximal pancreas, but the distal pancreas showed a significant increase of acini for positive cleaved-caspase 3 staining on day $3 \mathrm{PO}$, consistent with widespread induction of apoptosis in this cell type at this time (see Figure $3 f$ and Figure 5). A week after the transection, some acinar cells remained positive for cleaved-caspase 3 (Figure $3 \mathrm{j}$ ), but in the $2^{\text {nd }}$ and $3^{\text {rd }}$ weeks there was little evidence of cleaved-caspase 3 (Figure $3 \mathrm{n}, 3 \mathrm{r}$ ).

A week after the transection, duct-like structures showed positive immunostaining for cytokeratine-7, which increased over the post-operatory until the $3^{\text {rd }}$ week (see Figure $3 \mathrm{~g}, 3 \mathrm{k}, 3 \mathrm{o}, 3 \mathrm{~s}$ ) while zimogen staining showed a decreased expression with time.

No differences for insulin immunostaining were observed among different groups in the proximal pancreas. The distal pancreas showed a significant increase in positive insulin staining in scattered single islet cells and small islet-clusters but some ducts also appeared to be budding in the second and third weeks after transection (see Morphometric results).

\section{Morphometric results}

No differences in the islet size between groups were found in the proximal pancreas. However, a significant decrease $(p<0.05)$ in the mean area of the islets and the mean islet diameter was observed in the distal pancreas in groups 1-3 compared to groups 0,4 and 5 (Table 1). When an empirical distinction was made between small islets (with a profile area of less than $1000 \mu \mathrm{m}^{2}$ ) and larger islets (over $1000 \mu \mathrm{m}^{2}$ ), we found an increase of up to three times in the number of small islets in the distal 
(splenic) pancreas in groups 1-3 compared to groups 0,4 and $5(p<0.05)$ (Figure 4). Globally, there were significantly $(p<0.05)$ more islets and islet-cell clusters $(<1000$ $\mu \mathrm{m}^{2}$ ) in the distal pancreas of treated groups (groups 0-3) than in non-treated groups (groups 4-5).

The apoptotic response was quantified by the positive expression of cleavedcaspase 3 in the distal and proximal pancreas. No significant differences were found in cleaved-caspase 3 expression of the proximal pancreas over the PO, while the distal pancreas showed a significant positive expression at the $3^{\text {rd }}$ day with a rapid decrease over the PO. In fact, the best-fit least square function was obtained with the inverse curve for the cleaved-caspase 3 expression in the distal pancreas $(r=0.775 ; p<0.05)$ (Figure 5).

\section{Discussion}

In recent years, pancreatic surgeons have shown a growing interest in RF techniques. RF devices can be used in pancreatic surgery to fulfil one of two aims: 1) to directly ablate unresectable pancreatic tumors as a cytoreductive therapy in order to palliate their symptoms or even improve the survival of these patients ${ }^{24,25}$ (i.e. Radiofrequency ablation of pancreatic tumors) or 2) as a stump closure method because of its ability to seal or obliterate pancreatic ducts or small vessels in an attempt to avoid fistula formation usually after distal pancreatectomy ${ }^{9,14}$ (i.e. radiofrequency-assisted transection of the pancreas). In fact, similar devices with similar principles have been employed for either aim in the liver and more recently in the pancreas. For example, similar or identical RF cool-tip electrodes have been employed for either radiofrequency ablation or radiofrequency-assisted transection of the liver $r^{7,26}$ and the pancreas ${ }^{14,24,27,28}$. However, RF pancreatic ablation has been repeatedly warned to be associated with severe necrotizing pancreatitis with lifethreatening haemorrhagic complications, among other critical and dangerous complications $^{15,27}$. Even though, recent experiences seem to have reduced these 
severe complications using some prophylactic measures to avoid severe acute pancreatitis (such as exocrine secretion inhibition or reducing the ablative area to the tumor) 24,28,29 or thermal damage of the duodenum (using a duodenal cooling) ${ }^{30}$, this technique should be considered with extreme caution because of these serious complications ${ }^{8}$.

In our study we performed RF pancreatic ablation and section of the whole murine pancreas at the level of the portal vein without any other measures to prevent acute pancreatitis and the animals were terminated at different postoperative times. To our knowledge this is the first time that a RF-assisted transection of the pancreas has been performed leaving the distal pancreas without any drainage of the main pancreatic duct. In the present study, all the animals tolerated the procedure well and a common and constant pattern with no signs of necrotizing pancreatitis or fistula formation was observed. Only three micro-abscesses were observed which could have been probably caused by a primary contamination. Similarly to other protocols of RF ablation of the pancreas or RF-assisted transection of the pancreas, in our study a temporal and clinically irrelevant increase of amylase was also described ${ }^{14,31}$. In line with these authors' ${ }^{14,32,33}$ we can speculate that this elevation could be a normal consequence of thermally induced coagulative necrosis and consequent cytolysis of the pancreatic margin, which are the first histological steps toward the formation of the mature fibrosis found during necropsy.

Our findings, in fact, are similar to an efficient ligation of the pancreatic stump. Ligation of the main pancreatic stump without anastomosis is actually an old technique of managing the remnant pancreas after PD. It was originally performed by Whipple and is rarely performed today mainly because of an excessive incidence of postoperative pancreatic fistula ${ }^{6}$ (which can reach $50 \%$ of cases) ${ }^{34}$. The last prospective randomized trial of occlusion with glue of the pancreatic duct versus pancreaticojejunostomy after PD showed a higher fistula rate after duct occlusion (17\% versus $5 \%)^{5}$. This study also suggested a possible higher risk of diabetes in occluded 
pancreatic ducts, even though it was not powered to study this variable. In fact, the low rate of permeability of pancreas anastomosis in clinical studies has never been linked to endocrine insufficiency at least in pancreatogastrostomy $y^{35,36}$.

Ultrastructural, inmunohistochemical and studies with transgenic mice have demonstrated that after correct experimental duct ligation, most of the acinar cells are rapidly and massively deleted by p53-dependent apoptosis by caspase activation with some pancreatic epithelial proliferation ${ }^{1,2,37-40}$ and beta-cell neogenesis by newly formed islet-like cell clusters ${ }^{21,41,42}$. In our study, all these histological signs were clearly demonstrated in the distal pancreas of RF transected pancreas in comparison to proximal pancreas and the pancreas of sham and control groups with appropriate inmunohistochemical staining.

As some authors have suggested, the extent of pancreatic acinar cell apoptosis have been shown to be inversely related to the severity of pancreatic injury, suggesting that induction of a rapid and massive acinar cell apoptosis is a protective action against acute pancreatitis ${ }^{42-45}$. Likewise, Girelli et al, ${ }^{25}$ interestingly observed that tumor-related obstruction of the main pancreatic duct may reduce the risk of acute pancreatitis after RF pancreatic ablation. Thus, the rapid and massive acinar cell deletion in our study after RF ablation and section of the whole pancreas may explain why this model does not lead to necrotizing pancreatitis. This method could also be useful as a closure method of the pancreatic stump without pancreas anastomosis after PD in certain circumstances which could avoid the most significant cause of morbidity and mortality after PD. Further studies should confirm these results before its clinical application.

\section{Study limitations}

1. The murine model has been used extensively to study regeneration, pancreatitis and endocrine function ${ }^{22}$ but the obvious difference between the size of the pancreatic duct and the pancreas itself between rats and humans may limit the model's applicability to pancreatic surgery. In spite of this 
limitation, the absence of clinical and macroscopic pancreatic leakage is encouraging to carry out this experimental model in larger animals (which is actually ongoing in our laboratory). Additionally, in our model were unable to usually identify the main pancreatic duct in both the transection and the distal pancreas. Likely, the complexity and variations in the distribution of the pancreatic ducts (as was described by Richard, et al. ${ }^{16}$ ) may explain these findings.

2. This study provides evidence about beta-cell neogenesis by newly formed isletlike cell clusters in the distal pancreas after RF transection of the pancreas but this animal model is not useful to study the endocrine function of the distal remnant pancreas (because the proximal pancreas remains undamaged).

3. In this study it was evaluated the combine effect of ablation and transection of the ablated pancreas. The isolated effect of either procedures is out of the scope of the study.

Acknowledgments: This research has been completely supported by grant TEC2011-27133-C02-02 from the Spanish Government and the European Economic Community. The authors also thank Trevor Willis for his language revision. 


\section{Legends}

Figure 1. Serum amylase level at different time points. ${ }^{*} p<0.05$

Figure 2. Transection area. a) The arrow indicates the area of transection macroscopically, leaving to the left and right the proximal and distal margin, respectively. b) Transection area in the $3^{\text {rd }}$ week after $\mathrm{RF}$ ablation and section of the pancreas (Group 3). Cn: Coagulative necrosis surrounded by slight fibrosis (F) and spared multinucleated giant cells (arrow).

Figure 3. Immunohistochemical and HE staining of the proximal pancreas of Group 3 (a-d), distal atrophic pancreas of the Group 0 (e-h), Group 1 (i-l), Group 2 (m-p) and Group 3 (q-t). Line C3 notes the decrease of positive nucleus of cleaved-caspase 3 inmunohistochemistry with the postoperatory period, while duct-like structures increase (CK7). Insuline immunohistochemistry (I) shows positive staining for all groups.

Figure 4. Cell count of small islets (scattered and islet-cell clusters $<1000 \mu \mathrm{m}^{2}$ ) and larger islets among groups. Statistical analysis showed significant increase of small islets in the distal (splenic) pancreas in groups 1,2 and 3 compared to groups 0,4 and 5. However, no significant differences were found in the proximal pancreas. ${ }^{*} p<0.05$.

Figure 5. Quantitative analysis of cleaved-caspase 3 in the proximal and distal pancreas. The figure shows non-linear (inverse) relationship between cleaved-caspase 3 expression of the distal pancreas and the post-operatory (Groups $0-3$ ), while no significant difference is observed on the proximal pancreas. Goodness of fit of the model is represented by a dashed line is $r=0.775(p<0.05)$. 


\section{REFERENCES}

1. Wada $M$, Doi R, Hosotani $R$, et al. Apoptosis of acinar cells in rat pancreatic duct ligation. J Gastroenterol. 1995;30:813-814.

2. Walker NI. Ultrastructure of the rat pancreas after experimental duct ligation. I. The role of apoptosis and intraepithelial macrophages in acinar cell deletion. Am J Pathol. 1987;126:439-451.

3. Suc B, Msika S, Fingerhut A, et al. Temporary fibrin glue occlusion of the main pancreatic duct in the prevention of intra-abdominal complications after pancreatic resection: prospective randomized trial. Ann Surg. 2003;237:57-65.

4. Di Carlo V, Chiesa R, Pontiroli AE, et al. Pancreatoduodenectomy with occlusion of the residual stump by Neoprene injection. World $J$ Surg. 1989;13:105-110; discussion 110-101.

5. Tran K, Van Eijck C, Di Carlo V, et al. Occlusion of the pancreatic duct versus pancreaticojejunostomy: a prospective randomized trial. Ann Surg. 2002;236:422-428; discussion 428.

6. Fromm D, Schwarz K. Ligation of the pancreatic duct during difficult operative circumstances. J Am Coll Surg. 2003;197:943-948.

7. Ahmed M, Brace CL, Lee FT, Jr., et al. Principles of and advances in percutaneous ablation. Radiology. 2011;258:351-369.

8. Siriwardena AK. Radiofrequency ablation for locally advanced cancer of the pancreas. JOP. 2006;7:1-4.

9. Blansfield JA, Rapp MM, Chokshi RJ, et al. Novel method of stump closure for distal pancreatectomy with a $75 \%$ reduction in pancreatic fistula rate. $J$ Gastrointest Surg. 2012;16:524-528.

10. Nagakawa $\mathrm{Y}$, Tsuchida A, Saito $\mathrm{H}$, et al. The VIO soft-coagulation system can prevent pancreatic fistula following pancreatectomy. J Hepatobiliary Pancreat Surg. 2008;15:359-365.

11. Burdio F, Navarro A, Berjano E, et al. A radiofrequency-assisted device for bloodless rapid transection of the liver: a comparative study in a pig liver model. Eur J Surg Oncol. 2008;34:599-605.

12. Navarro A, Burdio F, Berjano EJ, et al. Laparoscopic blood-saving liver resection using a new radiofrequency-assisted device: preliminary report of an in vivo study with pig liver. Surg Endosc. 2008;22:1384-1391. 
13. Rios JS, Zalabardo JM, Burdio F, et al. Single instrument for hemostatic control in laparoscopic partial nephrectomy in a porcine model without renal vascular clamping. J Endourol. 2011;25:1005-1011.

14. Dorcaratto D, Burdio F, Fondevila D, et al. Radiofrequency is a secure and effective method for pancreatic transection in laparoscopic distal pancreatectomy: results of a randomized, controlled trial in an experimental model. Surg Endosc. 2013;27:3710-3719.

15. Elias D, Baton $O$, Sideris $L$, et al. Necrotizing pancreatitis after radiofrequency destruction of pancreatic tumours. Eur J Surg Oncol. 2004;30:85-87.

16. Richards C, Fitzgerald PJ, Carol B, et al. Segmental Division of the Rat Pancreas for Experimental Procedures. Lab Invest. 1964;13:1303-1321.

17. Bouwens L. Transdifferentiation versus stem cell hypothesis for the regeneration of islet beta-cells in the pancreas. Microsc Res Tech.1998;43:332336.

18. Fanjul M, Gmyr V, Sengenes C, et al. Evidence for epithelial-mesenchymal transition in adult human pancreatic exocrine cells. $J$ Histochem Cytochem. 2010;58:807-823.

19. Crawford HC, Scoggins CR, Washington MK, et al. Matrix metalloproteinase-7 is expressed by pancreatic cancer precursors and regulates acinar-to-ductal metaplasia in exocrine pancreas. J Clin Invest. 2002;109:1437-1444.

20. Elayat AA, El-Naggar MM, Tahir M. An immunocytochemical and morphometric study of the rat pancreatic islets. $J$ Anat. 1995;186:629-637.

21. Wang RN, Kloppel G, Bouwens L. Duct- to islet-cell differentiation and islet growth in the pancreas of duct-ligated adult rats. Diabetologia. 1995;38:14051411.

22. Hartig SM. Basic image analysis and manipulation in ImageJ. Curr Protoc Mol Biol. Vol Chapter 142013:Unit14 15.

23. Vert G, Chory J. Downstream nuclear events in brassinosteroid signalling. Nature. 2006;441:96-100.

24. Spiliotis JD, Datsis AC, Michalopoulos NV, et al. Radiofrequency ablation combined with palliative surgery may prolong survival of patients with advanced cancer of the pancreas. Langenbecks Arch Surg. 2007;392:55-60.

25. Girelli R, Frigerio I, Salvia R, et al. Feasibility and safety of radiofrequency ablation for locally advanced pancreatic cancer. Br J Surg. 2010;97:220-225.

26. Weber JC, Navarra G, Jiao LR, et al. New technique for liver resection using heat coagulative necrosis. Ann Surg. 2002;236:560-563. 
27. Wu $\mathrm{Y}$, Tang $\mathrm{Z}$, Fang $\mathrm{H}$, et al. High operative risk of cool-tip radiofrequency ablation for unresectable pancreatic head cancer. J Surg Oncol. 2006;94:392395.

28. Hadjicostas $\mathrm{P}$, Malakounides $\mathrm{N}$, Varianos $\mathrm{C}$, et al. Radiofrequency ablation in pancreatic cancer. HPB. 2006;8:61-64.

29. Girelli R, Frigerio I, Giardino A, et al. Results of 100 pancreatic radiofrequency ablations in the context of a multimodal strategy for stage III ductal adenocarcinoma. Langenbecks Arch Surg. 2013;398:63-69.

30. Fegrachi S, Molenaar IQ, Klaessens JH, et al. Radiofrequency ablation of the pancreas with and without intraluminal duodenal cooling in a porcine model. $J$ Surg Res. 2013;184:867-872.

31. Goldberg SN, Mallery S, Gazelle GS, et al. EUS-guided radiofrequency ablation in the pancreas: results in a porcine model. Gastrointest Endosc. 1999;50:392401.

32. Truty MJ, Sawyer MD, Que FG. Decreasing pancreatic leak after distal pancreatectomy: saline-coupled radiofrequency ablation in a porcine model. $J$ Gastrointest Surg. 2007;11:998-1007.

33. Kitagawa H, Ohta T, Tani T, et al. Nonclosure technique with saline-coupled bipolar electrocautery in management of the cut surface after distal pancreatectomy. J Hepatobiliary Pancreat Surg. 2008;15:377-383.

34. Aston SJ, Longmire WP, Jr. Management of the pancreas after pancreaticoduodenectomy. Ann Surg. 1974;179:322-327.

35. Pessaux $\mathrm{P}$, Aube $\mathrm{C}$, Lebigot $\mathrm{J}$, et al. Permeability and functionality of pancreaticogastrostomy after pancreaticoduodenectomy with dynamic magnetic resonance pancreatography after secretin stimulation. J Am Coll Surg. 2002;194:454-462.

36. Lemaire E, O'toole D, Sauvanet A, et al. Functional and morphological changes in the pancreatic remnant following pancreaticoduodenectomy with pancreaticogastric anastomosis. Br J Surg. 2000;87:434-438.

37. Walker NI, Winterford CM, Kerr JF. Ultrastructure of the rat pancreas after experimental duct ligation. II. Duct and stromal cell proliferation, differentiation, and deletion. Pancreas. 1992;7:420-434.

38. Scoggins CR, Meszoely IM, Wada M, et al. p53-dependent acinar cell apoptosis triggers epithelial proliferation in duct-ligated murine pancreas. Am J Physiol Gastrointest Liver Physiol. 2000;279:G827-836. 
39. Schuler M, Bossy-Wetzel E, Goldstein JC, et al. p53 induces apoptosis by caspase activation through mitochondrial cytochrome c release. $J$ Biol Chem. 2000;275:7337-7342.

40. Watanabe S, Abe K, Anbo Y, et al. Changes in the mouse exocrine pancreas after pancreatic duct ligation: a qualitative and quantitative histological study. Arch Histol Cytol. 1995;58:365-374.

41. Li M, Miyagawa J, Moriwaki M, et al. Analysis of expression profiles of isletassociated transcription and growth factors during beta-cell neogenesis from duct cells in partially duct-ligated mice. Pancreas. 2003;27:345-355.

42. Criscimanna A, Speicher JA, Houshmand G, et al. Duct cells contribute to regeneration of endocrine and acinar cells following pancreatic damage in adult mice. Gastroenterology. 2011;141:1451-1462, 1462 e1451-1456.

43. Iovanna JL. Redifferentiation and apoptosis of pancreatic cells during acute pancreatitis. Int J Pancreatol. 1996;20:77-84.

44. Bhatia M. Apoptosis versus necrosis in acute pancreatitis. Am J Physiol Gastrointest Liver Physiol. 2004;286:G189-196.

45. Kaiser AM, Saluja AK, Sengupta A, et al. Relationship between severity, necrosis, and apoptosis in five models of experimental acute pancreatitis. Am J Physiol. 1995;269:C1295-1304. 
Table 1. Mean islet area (A) and mean islet diameter (D) in proximal pancreas (duodenal lobe) and the distal pancreas (splenic lobe). Values are mean \pm SD. Significant difference $(\mathrm{p}<0.05)$ between groups $1-3$ and groups $0,4,5$.

\begin{tabular}{|c|l|c|c|c|c|c|c|}
\hline \multirow{2}{*}{\multicolumn{2}{|c|}{ Pancreas }} & \multicolumn{5}{|c|}{ Group } \\
\cline { 3 - 8 } & 0 & 1 & 2 & 3 & 4 (control) & 5 (Sham) \\
\hline $\mathrm{A}\left(\mu \mathrm{m}^{2}\right)$ & Proximal & $599 \pm 172$ & $517 \pm 168$ & $1371 \pm 488$ & $1061 \pm 339$ & $640 \pm 164$ & $1805 \pm 558$ \\
\cline { 2 - 8 } & Distal & $751 \pm 252$ & $320 \pm 117^{*}$ & $339 \pm 88^{*}$ & $452 \pm 158^{*}$ & $850 \pm 211$ & $1142 \pm 257$ \\
\hline $\mathrm{D}(\mu)$ & Proximal & $69 \pm 10$ & $63 \pm 10$ & $99 \pm 15$ & $88 \pm 17$ & $70 \pm 9$ & $118 \pm 23$ \\
\cline { 2 - 8 } & Distal & $63 \pm 13$ & $38 \pm 5^{*}$ & $45 \pm 5^{*}$ & $44 \pm 6^{*}$ & $83 \pm 11$ & $94 \pm 13$ \\
\hline
\end{tabular}




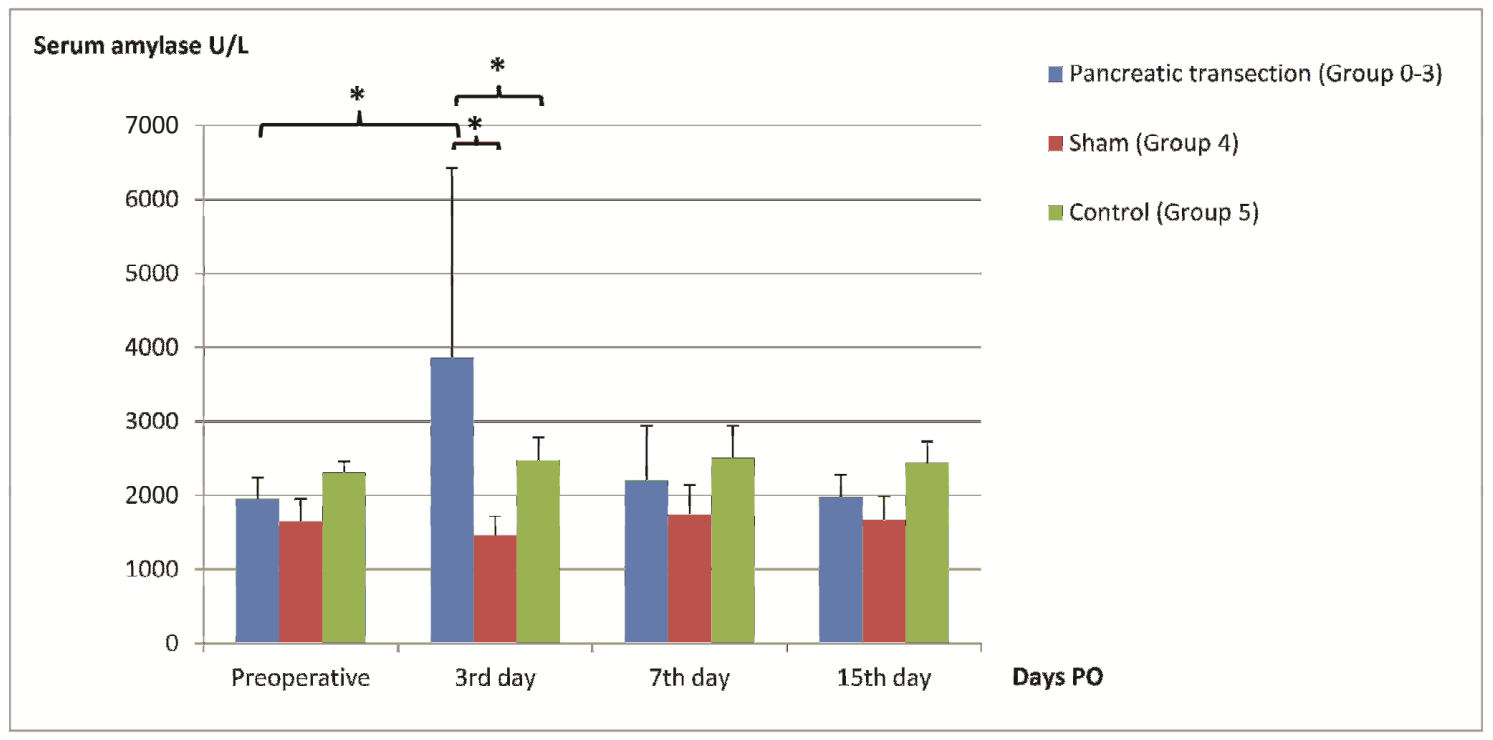

Figure 1. Serum amylase level at different time points. ${ }^{*} \mathrm{p}<0.05$ 

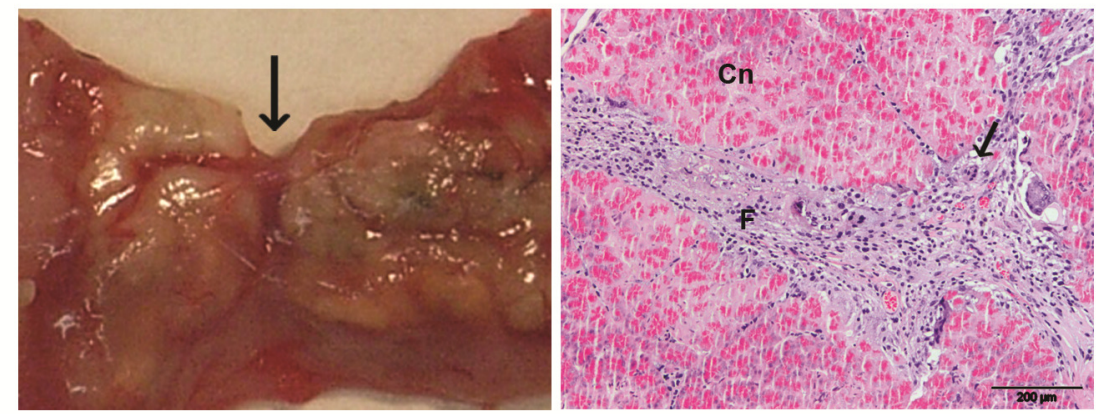

Figure 2. Transection area. a) The arrow indicates the area of transection macroscopically, leaving to the left and right the proximal and distal margin, respectively. b) Transection area in the $3^{\text {rd }}$ week after RF ablation and section of the pancreas (Group 3). Cn: Coagulative necrosis surrounded by slight fibrosis (F) and spared multinucleated giant cells (arrow). 


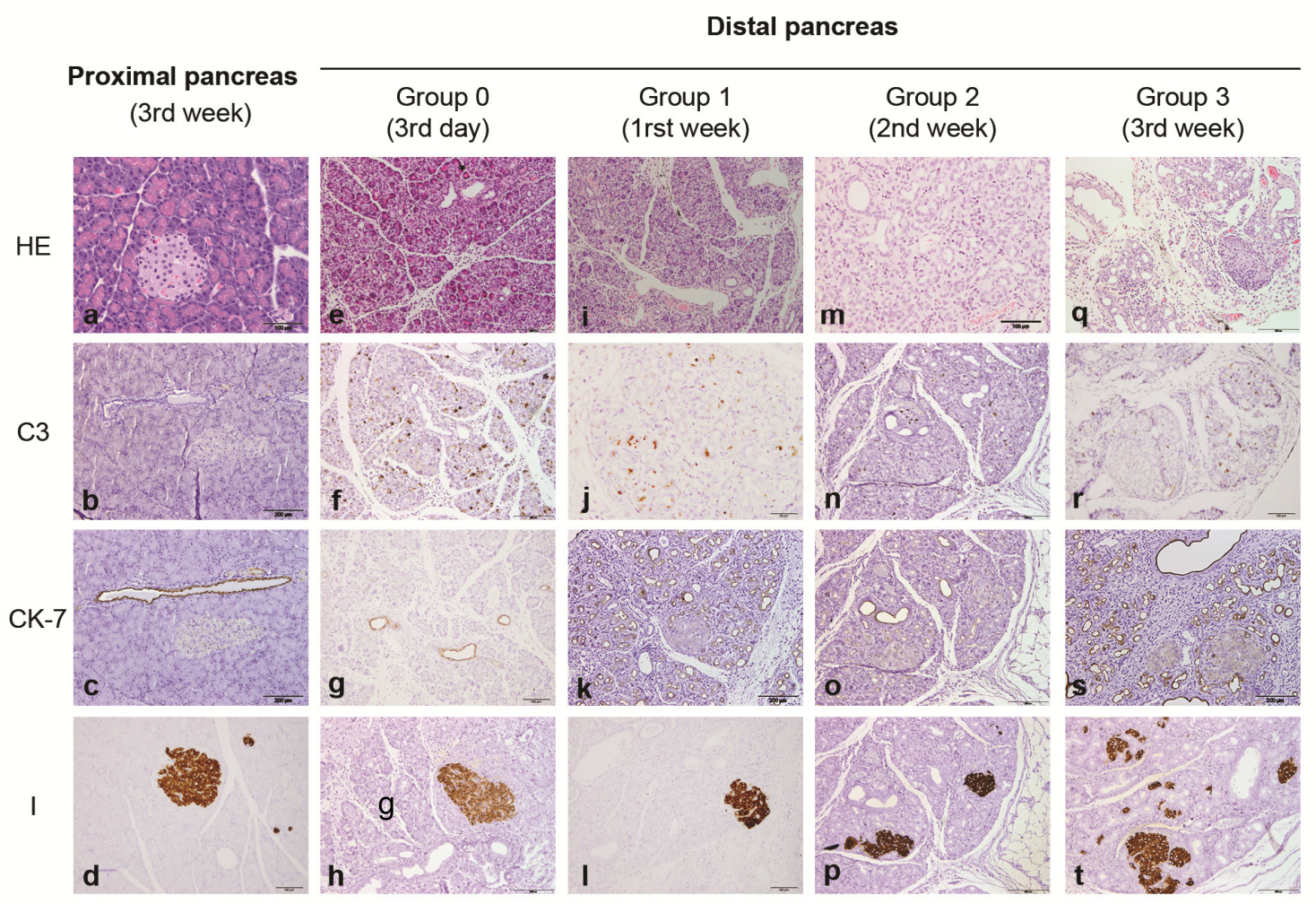

Figure 3. Immunohistochemical and HE staining of the proximal pancreas of Group 3 (a-d), distal atrophic pancreas of the Group 0 (e-h), Group 1 (i-l), Group 2 (m-p) and Group 3 (q-t). Line C3 notes the decrease of positive nucleus of cleaved-caspase 3 inmunohistochemistry with the postoperatory period, while duct-like structures increase (CK7). Insuline immunohistochemistry (I) shows positive staining for all groups. 

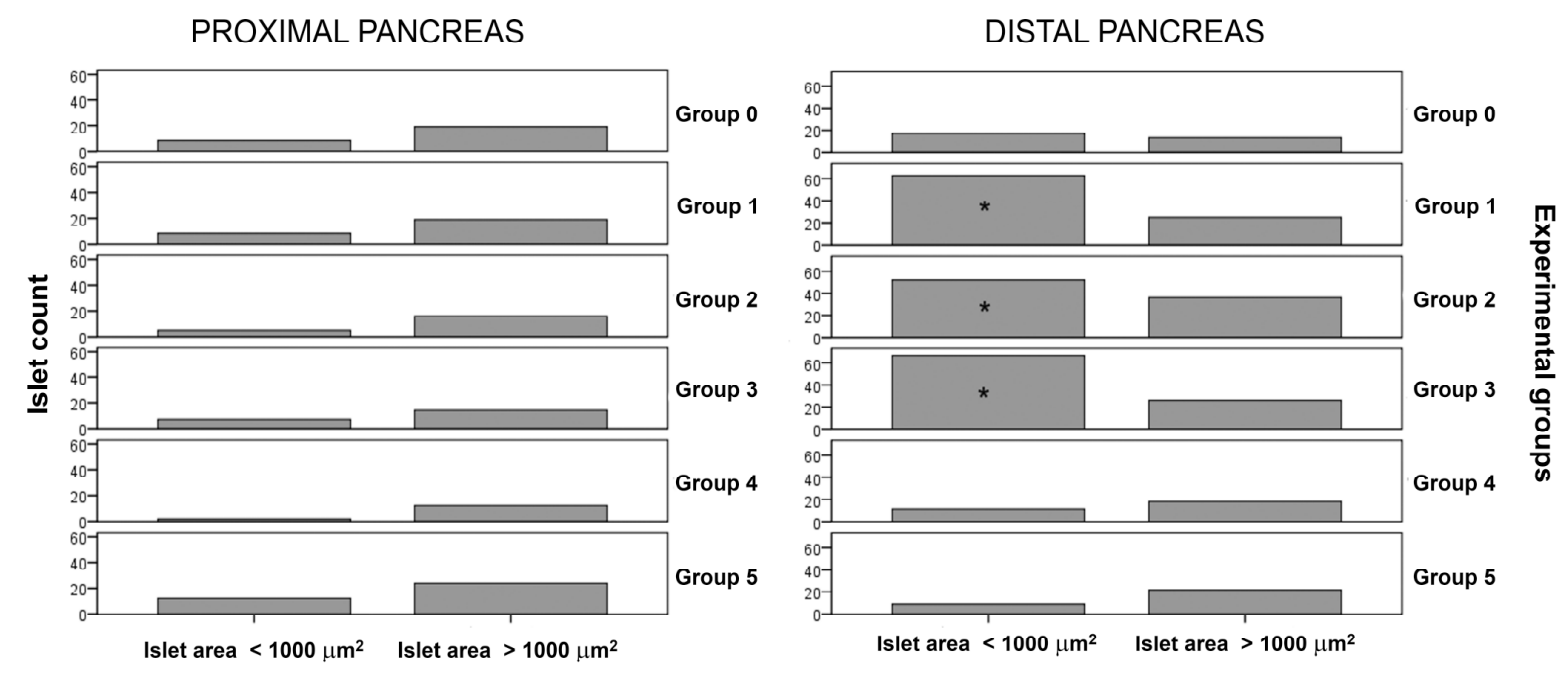

Figure 4. Cell count of small islets (scattered and islet-cell clusters $<1000 \mu \mathrm{m}^{2}$ ) and larger islets among groups. Statistical analysis showed significant increase of small islets in the distal (splenic) pancreas in groups 1, 2 and 3 compared to groups 0,4 and 5. However, no significant differences were found in the proximal pancreas. ${ }^{*} \mathrm{p}<0.05$. 


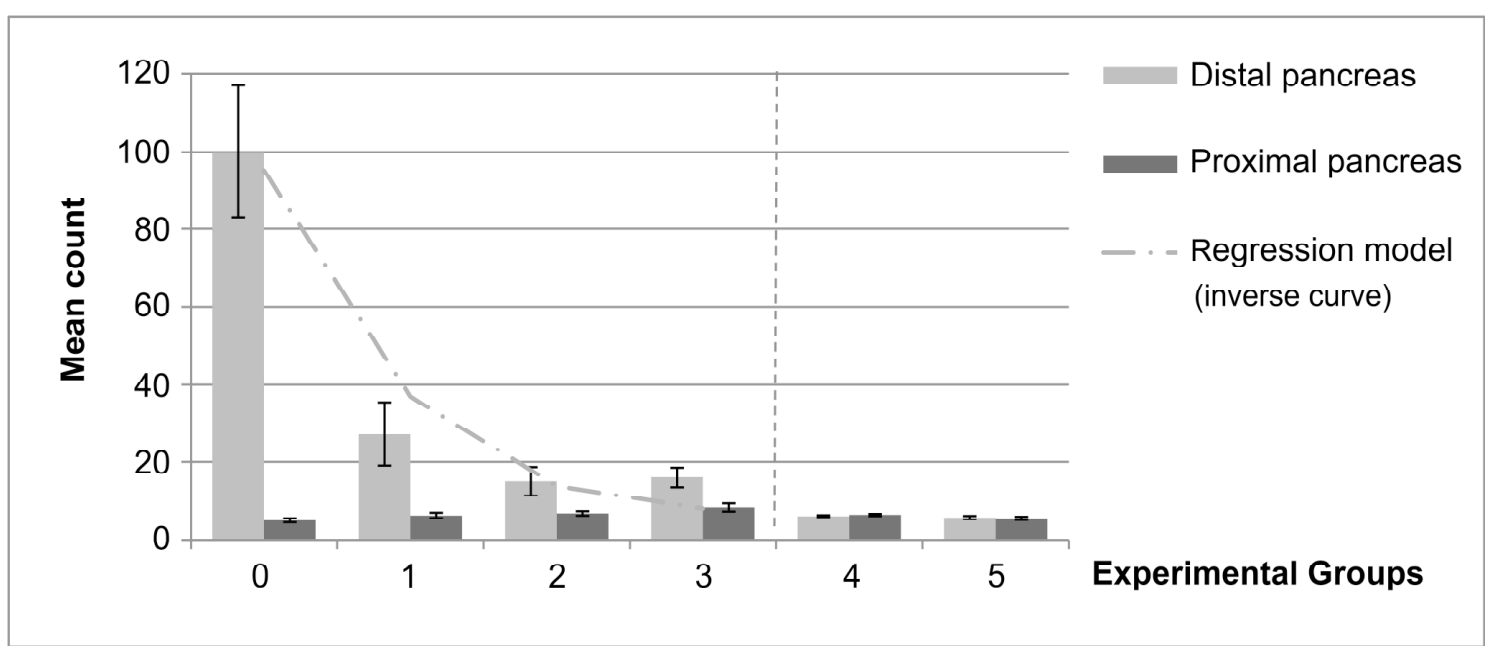

Figure 5. Quantitative analysis of cleaved-caspase 3 in the proximal and distal pancreas. The figure shows non-linear (inverse) relationship between cleaved-caspase 3 expression of the distal pancreas and the post-operatory (Groups 0-3), while no significant difference is observed on the proximal pancreas. Goodness of fit of the model is represented by a dashed line is $\mathrm{r}=0.775(\mathrm{p}<0.05)$. 